\title{
Incidence and Risk Factors of Relapse After Elective Electrical Cardioversion for Atrial Fibrillation: a Protocol for Systematic Review and Meta-analysis
}

\author{
Mazou Temgoua ( $\nabla$ neurotemgoua@yahoo.fr) \\ University of Yaounde I: Universite de Yaounde I https://orcid.org/0000-0002-2476-3550 \\ Jerome Boombhi
}

University of Yaounde I: Universite de Yaounde I

Joel Noutakdie Tochie

University of Yaounde I: Universite de Yaounde I

Clovis Nkoke

University of Buea

\section{Cedric Tsinda}

University of Yaounde I: Universite de Yaounde I

Alain Menanga

University of Yaounde I: Universite de Yaounde I

Samuel Kingue

University of Yaounde I: Universite de Yaounde I

\section{Protocol}

Keywords: Atrial Fibrillation (AF), electrical cardioversion, risk factors, systematic review

Posted Date: December 17th, 2020

DOl: https://doi.org/10.21203/rs.3.rs-129186/v1

License: (9) This work is licensed under a Creative Commons Attribution 4.0 International License.

Read Full License 


\section{Abstract}

\section{Background}

Atrial Fibrillation (AF) is the most frequent cardiac arrhythmia and it is associated with life-threatening complications such as hemodynamic instability and thromboembolic events. Electrical cardioversion remains its mainstay of treatment and can be performed either urgently or electively. Relapse after electrical cardioversion has been reported by several primary studies with divergent results, but no detailed summary exists for a critical appraisal of its global incidence and risk factors. Therefore, we propose the first systematic review and meta-analysis to synthesize the existing data on the global incidence and risk factors of relapse after electrical cardioversion for AF.

\section{Methods}

We will include case-control and cohort studies reporting on the incidence and risk factors of relapse after electrical cardioversion for atrial fibrillation. Electronic databases including PubMed, Embase, WHO Global Health Library and Web of Science will be searched for relevant records published between January 01,2000 , and December 15, 2020. Pairs of independents reviewers will perform study selection and data extraction, as well as an assessment of the methodological quality of included studies. Appropriate meta-analysis will then be used to pool studies judged to be clinically homogenous. Egger's test and funnel plots will be used to detect publication bias. Findings will be reported and compared by the human development level of countries.

\section{Results}

This systematic review and meta-analysis to synthesize the existing data on the global incidence and risk factors of relapse after electrical cardioversion for $\mathrm{AF}$

\section{Conclusion}

This review is expected to provide relevant data to help in evaluating the burden and risk factors of relapse after electrical cardioversion for atrial fibrillation. The overall findings of this research will be published in a peer-reviewed journal.

\section{Systematic Review Registration}

The protocol has been registered in Prospero with this registration number: CRD42020209301

\section{Background}

Atrial Fibrillation (AF) is a supraventricular tachyarrhythmia characterized by uncoordinated atrial activation with consequent deterioration of atrial mechanical function(1). The two major consequences are hemodynamic instability and thromboembolic events. AF is the most common sustained cardiac 
rhythm disturbance which increases with aging (1); observed in $3 \%$ of adults aged 20 years and above (2) (3). More representative regional figures report prevalence rates varying between $1 \%$ and $4 \%$ in Australia, Europe and the USA and an estimated prevalence varying between $0.49 \%$ and $1.9 \%$ in Asia. AF prevalence is highest in the Caucasian race. In Western Europe, Australia and North America $70 \%$ of people with AF are aged $>65$ years, whereas the mean age of AF patients in other geographical locations is often lower(4). AF can be classified as a first-detected episode, whether or not it is symptomatic or self-limiting. This implies that there can be uncertainty about the duration of the episode and previous undetected episodes. When a patient has had two or more episodes, AF is considered recurrent. Once terminated, recurrent AF is categorized as paroxysmal, and when sustained, persistent. In the latter case, termination by pharmacological therapy or electrical cardioversion does not change its terminology. Persistent AF (> 7 days) can be either the first presentation or a culmination of recurrent episodes of paroxysmal AF. Persistent AF includes cases of long-standing AF (greater than one year), in which cardioversion is not indicated or has not been attempted, usually leading to long-lasting AF (1). Management of AF includes anticoagulation according to the $\mathrm{CHA}_{2} \mathrm{DS}_{2}$-VASc score, heart rate regulation and cardiac rhythm control strategies (1)(5). One of the main means to control the cardiac rhythm during AF is through electrical cardioversion or direct-current cardioversion. This entails an electrical shock synchronized with the intrinsic activity of the heart(1). The rate of relapse after elective cardioversion is quite high and reported between 40 to $50 \%(6)$ and is influenced by the duration of AF, advanced age, female gender, tobacco abuse, chronic alcoholism, obesity,hypertension, obstructive sleep apnoea, diabetes mellitus, chronic obstructive pulmonary disease, renal impairment, hyperthyroidism, structural heart disease, left atrial size, heart failure and C-reactive protein (CRP) level(7)(8)(9).

Although the prevalence/incidence and risk factors of relapse after electrical cardioversion for AF has been widely reported by several isolated primary studies illustrating controversial findings, no detailed and comprehensive overview using rigorous methodological procedure has been carried out to date to scale up the evidence to enable decision making. Therefore, we propose the first systematic review and meta-analysis to critically synthesize the existing data on the global incidence and risk factors of relapse after electric cardioversion for atrial fibrillation.

\section{Review question}

What are the global incidence and the risk factors of relapse after electrical cardioversion for atrial fibrillation?

\section{Objective}

The systematic review and meta-analysis aim to determine the incidence and risk factors of relapse after electric cardioversion for atrial fibrillation

\section{Methods}


This review is reported in accordance with the Preferred Reporting Items for Systematic Review and MetaAnalysis Protocols 2015 Guidelines(10) applicable to observational studies. This is illustrated in more detail in online additional file 1.

\section{Eligibility criteria}

\section{Types of studies}

We will include case-control and cohort studies. Commentaries, editorials, letters and reviews will not be considered. Studies with inaccessible full-texts either online or after contacting its corresponding author will be excluded.

\section{Types of patients}

We will consider studies including adults and children.

\section{Type of study settings}

We will consider hospital-based studies in both rural and urban areas.

\section{Types of outcomes}

The incidence of relapse after elective cardioversion for AF will be defined as the number of individuals who developed a new episode of AF after a planned electric shock between January 01, 2000, and December 15, 2020. Risk factors will be derived from primary reported data. Data on pharmacological cardioversion and urgent cardioversion procedure will be excluded.

\section{Search strategy for identifying relevant studies}

We will search the following bibliographic databases: PubMed, Embase, WHO Global Health Library and Web of Science for relevant records published in English or French between January 01, 2000, and September 15, 2020. The designed search strategy for PubMed using both text words and medical subject heading (meSH) terms related to electric cardioversion, direct current cardioversion, electric shock, atrial fibrillation, relapse, recurrence, failure is provided in Table 1. This search strategy will be adapted to fit other databases.

\section{Searching for other sources}

We will scan the references of all relevant articles for additional data sources missed during our search strategy, and their full-texts will be sorted. Citations of important reviews will also be scanned. Lastly, the search strategy will extend to include grey literature from conference proceedings, book chapters, theses, government and non- governmental organizations' reports.

\section{Selection of studies for inclusion in the review}


Two reviewers (MNT and JNT) will independently evaluate the records obtained from the searching process, with the aid of an evaluation form to ensure a reliable application of the selection criteria. These reviewers will screen the titles and abstracts of records obtained. Next, the full-texts of potentially eligible articles will be retrieved by at least one author. The two reviewers will independently review the full text of each potentially eligible article, compare their findings and resolve any discordance by the arbitration of a third author $(\mathrm{CN})$. For duplicates articles, only the study reporting the largest sample size will be considered.

\section{Data extraction and management}

A data extraction form will be used by two pairs of independent reviewers (MNT, JNT, CN and CT), to collect information on the last name of the first author, year of publication, region and country of recruitment, human development index ranking of country economic level, study area (rural vs. urban), period of participants' inclusion, study setting(cardiology department, emergency unit, intensive care unit), age groups (children, adult, elders), number of sites, study design, mean or median age, sampling method(probabilistic sampling versus non probabilistic sampling), sample size, number of cases of AF, types of AF (paroxysmal or persistent), timing of data collection (prospective or retrospective), male proportion, specific characteristics of the study population (cardiomyopathy, coronary heart disease, congenital heart disease, valvular heart disease or any other specific condition), incidence rate of relapse, risk factors of relapse. For multicenter studies conducted in different countries, the incidence and risk factors will be reported separately for the individual countries.Countries of recruitment of participants will be categorized according to WHO regional classification: Africa, The Americas, South-East Asia, Europe, Eastern Mediterranean, and Western Pacific.

\section{Data synthesis and analysis}

After data collection, a meta- analysis will be conducted where there will be clinical homogeneity based on the profile of the population. Unadjusted SE, the incidence for the study-specific estimates will be resumed based on the crude information of the numerators, and denominators provided by each study. Sub-group analyses will be performed by separate pooling of studies conducted on specific groups of patients like cardiomyopathy, coronary heart disease,congenital heart disease andvalvular heart disease. To maintain the effect of studies with extremely small or large estimates on the overall estimate to a minimum, the variance of the study-specific incidence/risk factors will be stabilized with the FreemanTukey double arcsine transformation 19 , before pooling the data using a random-effects meta-analysis model. Heterogeneity will be assessed using the $\chi 2$ test on Cochrane's $Q$ statistic and quantified by calculating $\mathrm{I}_{2}(11)$.Values of $25 \%, 50 \%$ and $75 \%$ for $\mathrm{I}_{2}$ will, respectively, represent low, medium and high heterogeneity. We will assess the presence of publication bias using funnel plots inspection and Egger's test(12). Where substantial heterogeneity will be detected, a meta-regression and sub-group analyses will be performed to investigate the possible sources of heterogeneity using the aforementioned variables and the study methodological quality. In case of substantial clinical heterogeneity, a narrative summary of our findings will be done. The inter-rater agreement for study inclusion between investigators will be 
assessed using Cohen's k coefficient(13).Data analyses will be done using the 'meta' package of the statistical software R (V.3.5.1, The R Foundation for Statistical Computing, Vienna, Austria).

\section{Presentation and reporting of results}

The study selection process will be summarized in a flow diagram. Quantitative data will be presented in evidence tables of individual studies as well as in summary tables and forest plots where appropriate. The quality scores and risk of bias for each eligible study will be reported accordingly. This may be tabulated and accompanied by narrative summaries.

\section{Conclusion}

This review is expected to provide relevant data to help in evaluating the burden and risk factors of relapse after electrical cardioversion for atrial fibrillation. The overall findings of this research will be published in a peer-reviewed journal.

\section{Abbreviations}

AF: Atrial Fibrillation

CRP: C Reactive Protein

MESH: Medical Subject Headings

USA: United State of America

WHO: World Health Organization

\section{Declarations}

\section{- Ethical Approval and Consent to participate :}

In this study, data will be collected directly from published articles available in the main databases and unpublished studies. Patients and the public are not involved in the development of this protocol.

\section{- Consent for publication :}

Not applicable

\section{- Availability of supporting data :}

Not applicable

\section{- Competing interests :}


Non declared

\section{- Funding :}

The authors have no specific grant to declare for this research from any funding agency in the public, commercial or not- for- profit sector.

\section{- Authors' contributions :}

MNT, JB and JNT had the idea, designed and conceived the protocol. MNT wrote the first draft of the manuscript. MNT, AM and SK are the guarantors of the review. All authors critically revised the methodology and intellectual content and approved the final version of this manuscript.

\section{- Acknowledgements :}

Not applicable

\section{References}

1. Committee Members, Fuster V, Rydén LE, Asinger RW, Cannom DS, Crijns HJ, et al. ACC/AHA/ESC Guidelines for the Management of Patients With Atrial Fibrillation: Executive Summary A Report of the American College of Cardiology/American Heart Association Task Force on Practice Guidelines and the European Society of Cardiology Committee for Practice Guidelines and Policy Conferences (Committee to Develop Guidelines for the Management of Patients With Atrial Fibrillation) Developed in Collaboration With the North American Society of Pacing and Electrophysiology. Circulation. 2001 Oct 23;104(17):2118-50.

2. Haim M, Hoshen M, Reges O, Rabi Y, Balicer R, Leibowitz M. Prospective National Study of the Prevalence, Incidence, Management and Outcome of a Large Contemporary Cohort of Patients With Incident Non-Valvular Atrial Fibrillation. Journal of the American Heart Association [Internet]. 2015 Jan 5 [cited 2020 Sep 6];4(1). Available from: https://www.ahajournals.org/doi/10.1161/JAHA.114.001486

3. Björck S, Palaszewski B, Friberg L, Bergfeldt L. Atrial Fibrillation, Stroke Risk, and Warfarin Therapy Revisited. Stroke. 2013 Nov;44(11):3103-8.

4. Zulkifly H, Lip GYH, Lane DA. Epidemiology of atrial fibrillation. International Journal of Clinical Practice. 2018 Mar;72(3):e13070.

5. Kirchhof P, Benussi S, Kotecha D, Ahlsson A, Atar D, Casadei B, et al. 2016 ESC Guidelines for the management of atrial fibrillation developed in collaboration with EACTS. European Heart Journal. 2016 Oct 7;37(38):2893-962.

6. Vizzardi E, Curnis A, Latini MG, Salghetti F, Rocco E, Lupi L, et al. Risk factors for atrial fibrillation recurrence. Journal of Cardiovascular Medicine. 2014 Mar;15(3):235-53. 
7. Ecker V, Knoery C, Rushworth G, Rudd I, Ortner A, Begley D, et al. A review of factors associated with maintenance of sinus rhythm after elective electrical cardioversion for atrial fibrillation. Clinical Cardiology. 2018 Jun;41(6):862-70.

8. Raitt MH, Volgman AS, Zoble RG, Charbonneau L, Padder FA, O'Hara GE, et al. Prediction of the recurrence of atrial fibrillation after cardioversion in the Atrial Fibrillation Follow-up Investigation of Rhythm Management (AFFIRM) study. American Heart Journal. 2006 Feb;151(2):390-6.

9. Loricchio ML, Cianfrocca C, Pasceri V, Bianconi L, Auriti A, Calo L, et al. Relation of C-Reactive Protein to Long-Term Risk of Recurrence of Atrial Fibrillation After Electrical Cardioversion. The American Journal of Cardiology. 2007 May;99(10):1421-4.

10. Moher D, Shamseer L, Clarke M, Ghersi D, Liberati A, Petticrew M, et al. Preferred reporting items for systematic review and meta-analysis protocols (PRISMA-P) 2015 statement. Systematic Reviews. 2015 Jan 1;4(1):1.

11. Huedo-Medina TB, Sánchez-Meca J, Marín-Martínez F, Botella J. Assessing heterogeneity in metaanalysis: Q statistic or I² index? Psychological Methods. 2006;11(2):193-206.

12. Egger M, Smith GD, Schneider M, Minder C. Bias in meta-analysis detected by a simple, graphical test. BMJ. 1997 Sep 13;315(7109):629-34.

13. McHugh ML. Interrater reliability: the kappa statistic. Biochemia Medica. 2012;276-82.

\section{Table}

Table 1

Search strategy on Pubmed

\begin{tabular}{|ll|}
\hline Search & Searchterms \\
\hline 1 & «Incidence» OR « new onset rate » \\
\hline 2 & "Relapse » OR « Recurence » OR \\
3 & «Risk factors » OR « associated factors » OR « determinants » OR « predictors » \\
\hline 4 & Atrial fibrillation \\
\hline 5 & " Electric cardioversion » OR « direct- current cardioversion » OR « electric shock » \\
\hline 6 & \# 1 AND 2 AND 3 AND 4 AND $5 \#$ \\
\hline
\end{tabular}

\section{Supplementary Files}

This is a list of supplementary files associated with this preprint. Click to download.

- PRISMAPchecklist.docx 